\title{
Importancia de formar lectores críticos en educación superior
}

\author{
Ruby Estella Mendoza Afanador \\ Licenciada en Ciencias de la Educación, Español-Inglés \\ Universidad Pedagógica y Tecnológica de Colombia UPTC, \\ Especialista en Pedagogía para el Desarrollo \\ del Aprendizaje Autónomo \\ Universidad Nacional Abierta y a Distancia UNAD, \\ Magíster en Lingüística Aplicada a la Enseñanza del Inglés como \\ Lengua Extranjera, Universidad de JAÉN, España. \\ Integrante del grupo de Investigación SIEK \\ rubye71@hotmail.com
}

\author{
Luz Nélida Molano Avendaño \\ Licenciada en Ciencias de la Educación, Español - Inglés \\ Universidad Pedagógica y Tecnológica de Colombia UPTC, \\ Especialista en Pedagogía de la Lengua Escrita \\ Universidad Santo Tomás, \\ Especialista en Evaluación Pedagógica, \\ Universidad Católica de Manizales \\ Integrante del grupo de Investigación SIEK
}

Artículo de reflexión recibido el 10 de enero de 2015; aceptado el 27 de abril de 2015.

Cómo citar este artículo:

Mendoza, R. E. y Molano. L.N. (2015). Importancia de formar lectores críticos en educación superior. Espiral, Revista de Docencia e Investigación, 5(1), 101-116.

\section{Resumen}

El presente artículo de investigación tiene como objetivo describir una experiencia pedagógica investigativa, relacionada con la identificación del nivel de lectura crítica en estudiantes de VI semestre de la Licenciatura en Educación Básica, adscrita a la Facultad de Estudios a Distancia de la UPTC. Para lo cual se inicia una discusión teórica sobre algunos postulados relacionados con habilidades del pensamiento, pensamiento crítico y lectura crítica de autores como Cassany, Frank Serafini, Lipman, Klooster y Cooper, entre otros. Para el análisis del nivel de lectura crítica de la población objeto de estudio se utilizó una metodología de investigación descriptiva, ya que da cuenta de los diferentes hallazgos que se generaron a lo largo del estudio. El principal instrumento de análisis fue una prueba diagnóstica en la cual se identificaron las competencias y las dimensiones de lectura crítica propuestas por el ICFES para las pruebas Saber Pro. Los resultados permitieron determinar que gran parte de los estudiantes que ingresan al programa no cuentan con las habilidades del pensamiento y competencias comunicativas necesarias para asumir el rol de un lector crítico en las diferentes áreas del conocimiento dentro de la cualificación como profesionales; por lo cual se hace necesario crear espacios académicos que conduzcan a la reflexión acerca de cómo se han llevado a cabo las prácticas lectoras en el aula, y así proponer alternativas de solución transversales a todas las áreas que permitan la formación de verdaderos lectores críticos, que se reflejen a través de la interpretación y producción de discursos en diferentes escenarios y contextos.

Palabras clave: habilidades del pensamiento, pensamiento crítico, lectura crítica, dimensiones y competencias, formación.

\begin{abstract}
This investigations paper is to describe an investigative teaching experience related to the identification of the level of critical reading of the students of sixth semester of the Bachelor in Basic Education that belongs to the Faculty of distance learning of the UPTC. It's started a theoretical discussion of some principles related to thinking skills, critical thinking and critical reading of authors as Cassany Frank Serafini, Lipman, Klooster and Cooper, among others. A descriptive methodology research was used to analyze the level of critical reading of the target population of study, and that accounts the different findings that were generated during this study. The main instrument of analysis was a diagnostic test in which the competences and dimensions of the critical reading proposed by the ICFES identified for the Saber Pro tests. The results allowed to determine that most of the students who join the program do not have the thinking and communication skills needed to assume the role of a critical reader in different areas of knowledge within the qualifications required for professionals so it is necessary to create academic spaces that lead to reflect on how the reading practices have been implemented in the classroom to propose alternative solutions that cross all the areas and allow the formation of truly critical readers that reflect through the interpretation and production of speeches in different settings and contexts.
\end{abstract}

Keywords: thinking skills, critical thinking, critical reading, dimensions and skills training.

\section{Introducción}

Una de las grandes preocupaciones de los docentes, en los diferentes niveles de educa- 
ción, es el grado de comprensión lectora de sus estudiantes, por lo cual muchas de las actividades que se plantean, tanto dentro como fuera del aula, están estrechamente ligadas a esta habilidad comunicativa; toda vez que el conocimiento en las áreas del saber se encuentra condensado en libros, documentos, revistas, blogs, artículos, páginas web especializadas, entre otros. No siempre el resultado de esta lectura es el esperado por el docente. ¿Pero qué expectativas tienen de sus estudiantes? Por lo general que no solo recuperen la información del texto, sino que estén en capacidad de dar una opinión crítica y reflexiva a través de la elaboración de un ensayo, un artículo, un comentario u otro producto que evidencie la apropiación del texto en todas sus dimensiones con argumentos sólidos y profundos.

Estudios realizados sobre los resultados de las pruebas Saber Pro, examen de Estado de Calidad de la Educación Superior, aplicado directamente por el ICFES (Instituto Colombiano para el Fomento de la Educación Superior), demuestran que la lectura crítica es uno de los elementos que incide directamente en el desempeño de los estudiantes en dichas pruebas. Es el caso de la investigación llevada a cabo por la Licenciatura en Etnoeducación, Universidad de la Guajira (2014), en la que determinó que con respecto a la lectura crítica los estudiantes presentan dificultades para ubicar información sobre eventos, actores, circunstancias, escenarios, momentos o fuentes de información; para reconocer la relación de un texto con otros textos; los puntos de vistas que circulan en un texto o situación de comunicación, entre otros. Con la propuesta investigativa de la Universidad Católica del Norte (2011), acerca de la lectura crítica en la Universidad de Medellín, se buscó reforzar el impacto o incidencia que tienen los enfoques teóricos y metodológicos de la lingüística textual y el análisis crítico del discurso en los procesos de cultura escrita, especialmente en lectura crí- tica; el estudio plantea un modelo de lectura crítica basado en la propuesta que hace Daniel Cassany en su libro Tras las líneas. Este modelo logró demostrar que los cursos basados en estos enfoques obtienen mejores resultados en el análisis e interpretación textual, contribuyendo así a la formación de lectores críticos e independientes.

La lectura es uno de los procesos más complejos a que se ve abocado el ser humano desde el inicio de su vida escolar, siendo la escuela la principal encargada de esta tarea, por lo que se espera que esta forme "lectores capaces de elegir o seleccionar sus lecturas, tener un punto de vista manifestado en una opinión, y seguir cultivándose a lo largo de toda la vida" (Vásquez, 2015, p. 61); es decir, que alcancen un nivel de lectura crítica que coadyuve a un desempeño de calidad en situaciones comunicativas no solo en la etapa escolar sino también en su vida profesional y personal.

Teniendo en cuenta los aspectos anteriormente enunciados, este documento muestra un ejercicio de lectura crítica basado en las dimensiones y competencias propuestas por el Ministerio de Educación Superior (MEN), para las pruebas Saber Pro. Esta evaluación diagnóstica se llevó a cabo con estudiantes de VI semestre de la Licenciatura en Educación Básica de la Facultad de Estudios a Distancia de la Universidad Pedagógica y Tecnológica de Colombia (UPTC), quienes en un $70 \%$ ingresan a la institución por convenios interinstitucionales con algunas escuelas normales superiores $y$, el 30\% restante, han desarrollado procesos formativos desde el primer semestre en el programa. Dicho estudio se presenta de la siguiente forma: primero se hace una breve discusión conceptual de aspectos relacionados con la lectura crítica, sus dimensiones y niveles; luego se realiza un análisis del trabajo investigativo, haciendo uso de una metodología de tipo descriptiva, para finalmente dar algunas conclusiones. 


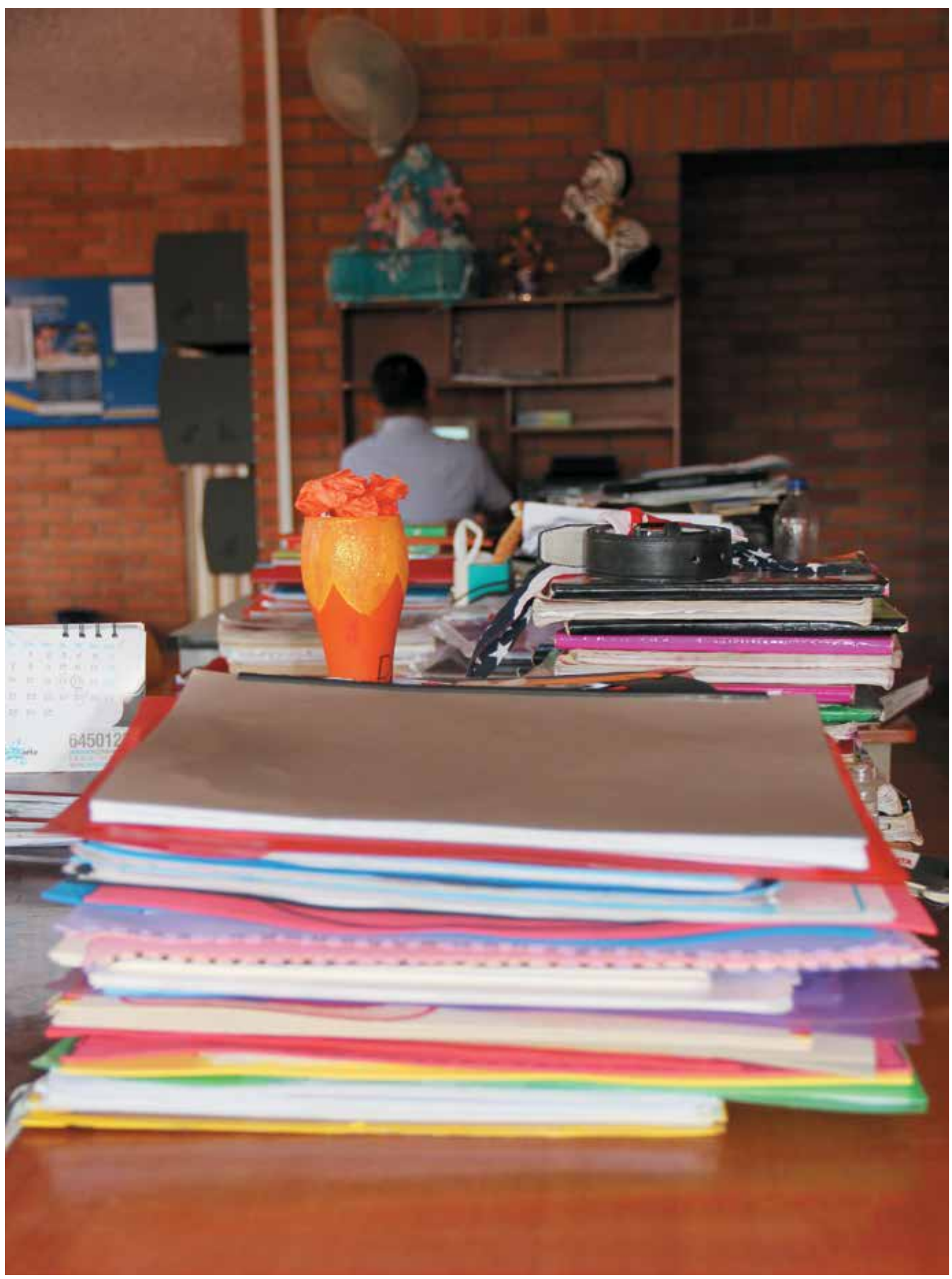




\section{Discusión conceptual}

\section{Pensamiento crítico}

Es necesario hacer referencia al pensamiento crítico como condición indispensable previa, para empezar a hablar de lectura crítica. Al respecto se pueden encontrar gran variedad de definiciones, pero en esta ocasión se quiere destacar autores como Giroux (1988), quien enfatiza dos características relevantes del pensamiento crítico: la primera hace referencia a que el lector no debe entender el conocimiento como un fin sino como un medio para problematizar constantemente los hechos y, la segun$\mathrm{da}$, que ningún conocimiento carece de intereses, normas y valores humanos subyacentes, por lo que resulta fundamental poder adoptar varios puntos de vista y desarrollar el pensamiento dialéctico. Por consiguiente, el pensamiento crítico permite desarrollar habilidades cognitivas y metacognitivas en el estudiante para aprender a pensar, a diferenciar puntos de vista, a crear argumentaciones y teorías que lo conduzcan a generar nuevos conocimientos $y$, a la vez, tomar decisiones con el fin de solucionar problemas de su contexto.

Por otro lado, Kanpool (1994) enuncia que la crítica es "el empoderamiento individual, en el sentido posmoderno, para analizar y sintetizar la cultura de la escuela y las propias y particulares circunstancias culturales". Aquí se puede apreciar que el pensamiento crítico es tomado como la apreciación personal que cada uno pueda construir en relación con los aspectos culturales, tanto de los espacios académicos como cotidianos en los que el sujeto se encuentra inmerso y, a partir de los cuales pueda elaborar discursos o argumentaciones que le permitan interactuar con propiedad y firmeza en situaciones de comunicación, donde sus ideas sean sólidas y den oportunidad a la reciprocidad de diálogos coherentes y claros que conduzcan a la toma de decisiones para lograr cambios positivos en una población determinada.
Klooster (2001) afirma que al hablar de crítica no se debe relacionar con memorización ni mucho menos conformarse con la simple comprensión del texto, tampoco lo es la creatividad ni la intuición. El autor presenta cinco rasgos que deben caracterizar al pensador crítico: el ser independiente, el estar informado, el ser cuestionador a través de preguntas o problemas que interesen al sujeto y que debe resolver, ser razonador en sus argumentos, tesis, pruebas, entre otros; y actuar como ser social para comparar, contrastar y compartir las ideas con los demás aunque al inicio el proceso sea individual.

Con base en los anteriores postulados propuestos por Klooster, la formación de personas críticas se hace imprescindible si se quiere lograr una sociedad justa y equitativa, donde las personas puedan actuar libremente, pero con responsabilidad. Por tal razón, es importante que en el aula de clase se propicien espacios de aprendizaje para que los estudiantes tengan la posibilidad de estar en contacto permanente con textos como la prensa impresa o digital, el cine, la pintura, la novela, anuncios publicitarios, entre otros que permitan el acercamiento a situaciones cotidianas como la guerra, la inequidad, la violencia, el desempleo, el desplazamiento y la pobreza, que son temas adecuados para el desarrollo de un pensamiento crítico; pero que infortunadamente no son muy frecuentes en la educación básica o superior, por cuanto el docente no tiene la preparación académica y metodológica requeridas para abordar y orientar este tipo de textos.

Lamentablemente se está impidiendo que los niños y jóvenes se formen como personas críticas para desempeñarse adecuadamente en un mundo globalizante, caracterizado por grandes cambios sociales y culturales, bajo el dominio de los medios masivos de comunicación, pese a los ingentes esfuerzos del Estado por lograr un cambio radical en la forma como se deben asumir tanto las lecturas académicas como las críticas. El desarrollo de habilidades 
de pensamiento y la conformación de un repertorio considerable de argumentaciones que deben caracterizar a un buen pensador crítico, se están quedando plasmadas en reflexiones de artículos y políticas educativas que no trascienden a la realidad de un país que requiere con urgencia la participación activa de sus habitantes, para contribuir de alguna forma en cambios significativos que propendan por una mejor calidad de vida donde los cimientos sean la paz, la convivencia, la tolerancia y la responsabilidad social.

\section{Lectura crítica}

Este tipo de lectura requiere del lector la suma de una serie de habilidades que le permitan identificar las intencionalidades del autor con respecto a una idea, un concepto, un saber, una ideología dentro de un contexto específico de comunicación, en palabras de Rodríguez (2007) "exige al lector elaborar un significado del texto involucrando, al mismo tiempo, la reconstrucción de la huella que el autor dejó en él". Pero para llegar a este grado de comprensión, es indispensable haber pasado primero por una serie de estadios que van desde la decodificación primaria, la reconstrucción global y parcial del texto, la identificación de significados explícitos e implícitos hasta el empleo consciente e inconsciente de estrategias metacognitivas de lectura que lo conviertan en un lector autónomo, crítico y reflexivo.

En educación superior este tipo de lectura, es el más solicitado por los docentes, pero se espera que los estudiantes ya tengan las competencias y habilidades suficientes para acercarse a los textos con una mirada crítica, de tal manera que se reconozcan aspectos de carácter textual, intertextual y extra textual, en otras palabras, que sean capaces de leer las líneas, entre líneas y detrás de las líneas del discurso, sin embargo, falta proporcionarles más herramientas y estrategias necesarias que lo conviertan en un lector crítico.
Leer las líneas es el primer paso para acceder al texto, aquí el lector reconoce palabras y frases con sus correspondientes significados, para posteriormente parafrasear y reconstruir el significado parcial y total del texto. "Las lecturas de primer nivel o literales son realizadas en el marco del diccionario o de los significados estables integrados a las estructuras superficiales del texto" (MEN, Lineamientos curriculares de Lengua Castellana (1990). Entre líneas requiere un nivel de comprensión más profundo del texto, el lector realiza inferencias y deducciones que no se encuentran explícitas en el texto como tal. Cooper (1990) citado por Maqueo (2006) afirma que las inferencias que hace un lector se basan en cierta medida en su información, experiencias y conocimientos previos y en las claves que proporciona el autor. Así pues, este tipo de lectura requiere de un lector más activo, que procesa y otorga significado al discurso impreso.

Pero cuando el lector es capaz de identificar la ideología y el contexto, social, político, económico, religioso que motivaron al autor, se dice que está leyendo detrás de las líneas o que ha adquirido un nivel de lectura crítica o literacidad. Cassany (2013) anota que la lengua es algo abstracto, contrario al discurso que sí existe como tal y que todos los discursos están situados, es decir, tienen un autor que a su vez pertenece a una comunidad, establece unas relaciones con ella, participa de los cambios de la sociedad, no es ajeno a lo que lo rodea, toma partido de lo que le afecta. Por lo tanto, para este autor, "los discursos neutros, objetivos o desinteresados, no existen".

Según Freire (1989) la lectura debe ser propuesta como un acto dinámico, no debe convertirse en un acto de memorización o de cumplimiento de una obligación, debe ser para los estudiantes una forma de encontrarse con otros mundos, otras realidades que le permitan aportar a su vivencia y convivencia. Así mismo, para Freire, la lectura es un acto eminentemente político, una herramienta para actuar en la 
sociedad, un instrumento para mejorar las condiciones de vida del aprendiz. En consecuencia un lector crítico debe defender sus ideas, pero respetar las del otro con tolerancia, de tal manera que la lectura que realice del texto pueda dar origen a nuevos discursos que nutran los ya existentes en un marco multicultural y divergente a través del cual se pueda construir ciudadanía.

El concepto de lectura crítica ha evolucionado con los años debido a los cambios en la sociedad, la educación y las necesidades del mundo posmoderno. El lector de ahora no es el lector de antes, requiere nuevas formas, estrategias, tipos y temas de lectura; sus necesidades son diferentes como los contextos en que se desenvuelve. La globalización, la circulación de la información a través de la red, el auge de las tecnologías de la información y la comunicación, entre otros exigen el desarrollo adicional de competencias tecnológicas, informáticas, comunicacionales para procesar, analizar y transferir la información, de tal manera que conlleve a la generación de nuevo conocimiento, no solo almacenar y reproducir sin sentido. Así mismo, ser crítico en relación con la lectura va más allá de realizar inferencias, hipótesis, identificar la intención del autor o realizar análisis de fondo y forma de una obra literaria. El mundo actual requiere un nivel de literacidad en el cual "el discurso no posee conocimiento en sí, sino que este emerge al entrar en contacto con los significados que aporta cada comunidad a través del lector" Cassany (2013, p. 82).

Frank Serafini (2003) diferencia tres perspectivas teórico-prácticas en la educación lectora: modernista, transaccional y crítica; para esta última expresa que el texto está situado sociohistóricamente, y es un artefacto cultural con propósitos y contexto social, histórico, político, cultural en el cual, además, es importante tener en cuenta aspectos de género, etnia, cultura, clase social, entre otros. Es así que el texto no debe ser visto como una reproducción de ideas y conocimientos sino como un ente vivo capaz de reflejar la sociedad en el cual está inmerso, y quien lo lee tiene la responsabilidad de construir significa- dos a través del desarrollo y puesta en marcha de una serie de competencias como la enciclopédica, semántica, analítica y crítica.

En consecuencia, desarrollar niveles de literacidad en los estudiantes requiere de un análisis crítico del discurso desde las propuestas de autores como Van Dijk (1989), quien a partir de su "lingüística del texto", propone unas categorías relacionadas con un nivel intratextual que tiene que ver con la presencia de microestructuras y macroestructuras que garantizan una coherencia y cohesión de tipo local; un nivel intertextual en el cual se relaciona el texto con otros textos, otras épocas, otras voces $y$, finalmente, un nivel extratextual o pragmático en el cual se identifican componentes ideológicos y políticos que están ocultos o presentes en el texto. No es recomendable desligar un nivel del otro ni abordarlo desde situaciones aisladas de comunicación, ya que el discurso sea oral o escrito es una actividad social por excelencia.

\section{Competencias de la lectura crítica}

De acuerdo con el Módulo de lectura crítica Saber Pro (2014), las pruebas están diseñadas para evaluar tres competencias básicas:

Identificar y entender los contenidos locales que conforman un texto. Esta competencia integra la capacidad de identificar y comprender los eventos, ideas, afirmaciones y demás elementos locales que componen un texto. En esta fase, la evaluación está orientada a la comprensión del significado de palabras, expresiones o frases que aparecen explícitamente en el texto. Es importante anotar que en ausencia de esta competencia no es posible contar con las dos siguientes.

Comprender cómo se articulan las partes de un texto para darle un sentido global. Esta competencia hace referencia a la capacidad de comprender cómo se relacionan semántica y formalmente los elementos locales que constituyen un texto, de manera que este adquiera un sentido global. De esta forma, las preguntas 


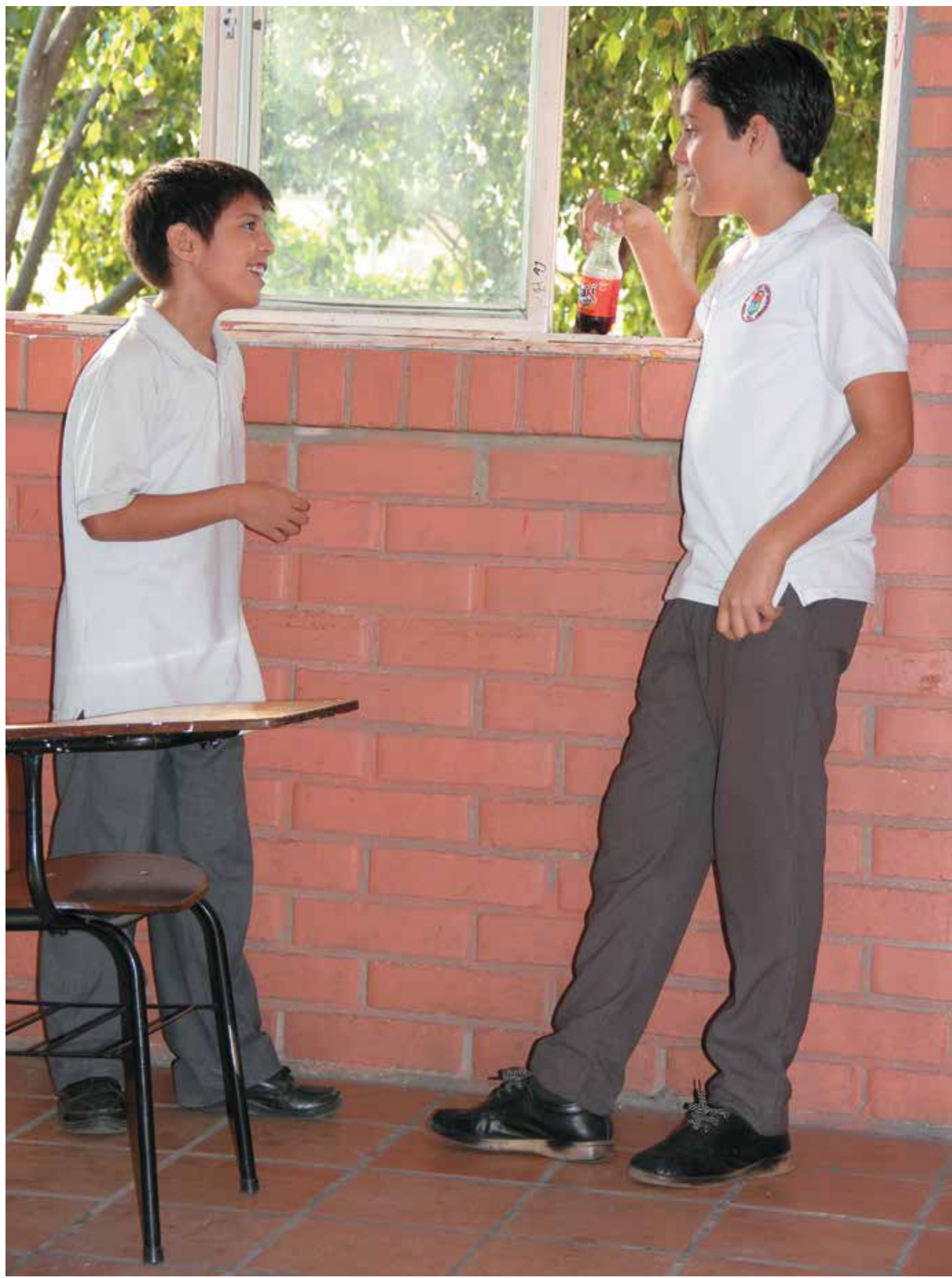


que la evalúan involucran elementos locales de un texto y exigen reconocer y comprender su articulación. Esta competencia es indispensable para que se pueda identificar la siguiente.

Reflexionar a partir de un texto y evaluar su contenido. Esta competencia hace alusión a la capacidad de enfrentar el texto críticamente. Por tal razón, incluye evaluar la validez de argumentos, advertir supuestos, derivar implicaciones, reconocer estrategias argumentativas y retóricas, relacionar los contenidos con variables contextuales, entre otros. Esta es la competencia esencialmente crítica, que requiere indiscutiblemente un adecuado de las dos competencias anteriores.

\section{Dimensiones de la lectura crítica}

Figura 1. Dimensiones de la lectura crítica

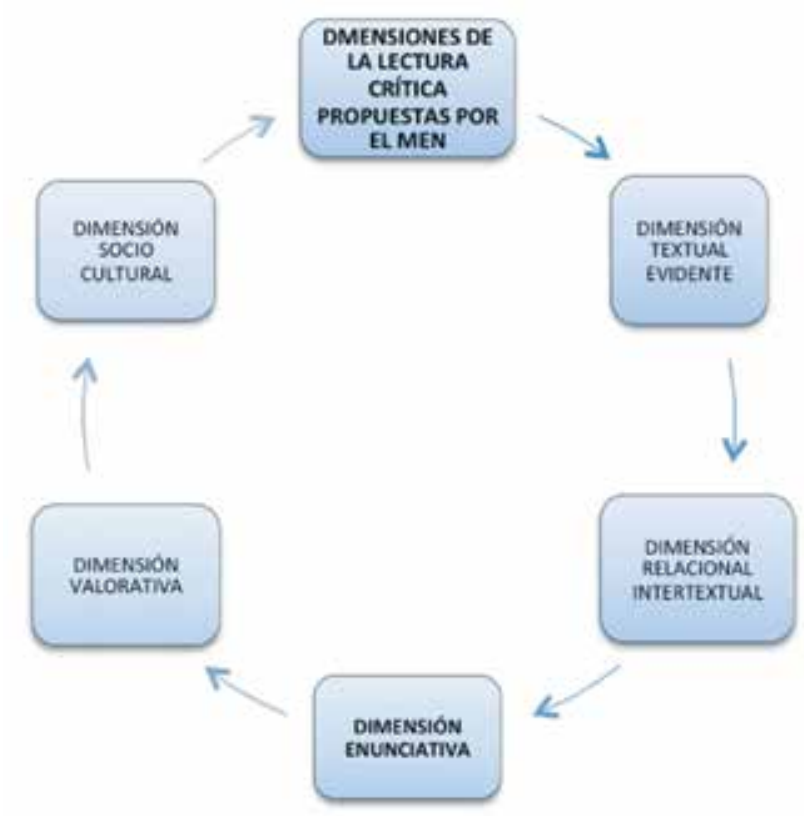

Fuente: autores.

Como se puede apreciar en la figura 1, la primera dimensión crítica según la propuesta del Ministerio de Educación Nacional es la textual evidente, la cual hace referencia a cómo el lector a través de procesos de ubicación y articulación de información, comprende el contenido de un texto, en otras palabras, realiza una primera tarea cognitiva de apropiación literal del texto, con el fin de conocer el contenido su- perficial y el contenido global de este. Luego, se presenta la dimensión intertextual, donde el lector avanza en la identificación de las relaciones que pueden ser reconocidas en el interior del texto y que hacen alusión tanto a aspectos sintácticos como semánticos. Como tercera dimensión, se encuentra la dimensión enunciativa y su principal función consiste en indagar por los enunciados que conforman el texto, en su relación con quiénes participan en la situación de comunicación (enunciadores) y las intenciones que se buscan de acuerdo con una audiencia específica. En el cuarto lugar, se encuentra la dimensión valorativa, que ya es una dimensión estrictamente crítica y hace uso de recursos pragmáticos (evaluados en la dimensión inmediatamente anterior) y críticos que le permiten al lector ahondar más en el texto, pero asumiendo una visión crítica del uso del lenguaje. Finalmente, en la quinta dimensión, que se convierte en la segunda dimensión propiamente crítica, por cuanto se hace más evidente el carácter sociocultural que siempre se le ha atribuido a la lectura al asimilarse a una práctica que se produce en el seno social, que genera construcciones simbólicas solidarias entre productores y lectores del texto y que ocasionan un efecto que puede definir el discurso a partir de la práctica o la práctica a partir del discurso (MEN, Módulo Lectura Crítica, 2014).

\section{Enfoque metodológico}

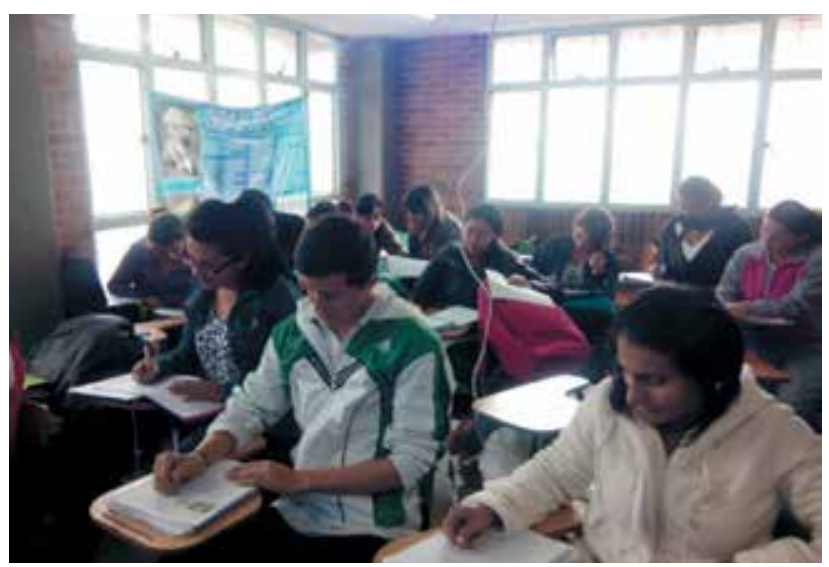

Fuente: los autores. 
Esta investigación es descriptiva, puesto que se enfoca en comprender y profundizar los fenómenos, prácticas desde la perspectiva de los participantes en un ambiente natural y en relación con el contexto (Sampieri, 2010). Así mismo, en cuanto que permite "describir un fenómeno o una situación, mediante el estudio del mismo en una circunstancia tempo-espacial determinada" (Ander-Egg, 1984, p. 84).

El principal objetivo de este trabajo se centra en describir el nivel de lectura crítica de los estudiantes de VI semestre de la Licenciatura en Educación Básica con Énfasis en Matemáticas, Humanidades y Lengua Castellana de la UPTC, a partir de las prácticas y experiencia personal de los participantes, así como de una revisión teórica sobre el tema. La muestra tomada fueron 36 estudiantes del Centro Regional de Educación a Distancia (CREAD), Tunja, quienes en su mayoría son egresados de escuelas normales, e ingresan por convenio a $\mathrm{V}$ semestre del Programa.

Para los propósitos de la investigación se aplicó una prueba de entrada, con el fin de medir las competencias de lectura crítica propuestas en las Pruebas Saber Pro, para establecer cómo los estudiantes identifican y entienden los contenidos locales del texto; de qué manera articulan las partes de un texto para darle un sentido global que los conduzca a la reflexión de la lectura y a evaluar su contenido; así mismo, se tuvieron en cuenta las dimensiones presentes en la lectura crítica, a saber: dimensión textual evidente, dimensión enunciativa, dimensión valorativa y dimensión sociocultural. A partir del análisis descriptivo de estas competencias y dimensiones se determinó el nivel de lectura crítica de la población objeto de estudio, para posteriormente proponer alternativas de solución.

\section{Análisis de resultados}

En esta investigación se aplicó una prueba, la cual está conformada por dos textos y cada uno de ellos contiene varias preguntas. A continuación se presenta el análisis de dicha prueba, primero por frecuencia y porcentajes y, posteriormente, por cada una de las preguntas, y en cada una de ellas se especifican las competencias.

\section{a. Análisis frecuencia y porcentaje global}

Tabla1. Quintil Análisis de la Prueba

\begin{tabular}{cccc}
\hline DIMENSIONES & FRECUENCIA & PORCENTAJE & $\begin{array}{c}\text { PORCENTAJE } \\
\text { ACUMULADO }\end{array}$ \\
\hline I & 6 & 17 & 17 \\
II & 19 & 53 & 60 \\
III & 10 & 28 & 88 \\
IV & 1 & 2 & 100 \\
V & 0 & 0 & 0 \\
\hline TOTAL & 36 & 100 & \\
\hline
\end{tabular}

La anterior tabla muestra la frecuencia y el porcentaje obtenido por los estudiantes en cada una de las dimensiones de lectura crítica, teniendo en cuenta que el nivel I corresponde a dimensión textual evidente, el II, dimensión relacional intertextual, III dimensión enunciativa, IV, dimensión valorativa y, V, dimensión sociocultural. Tenemos que:

Figura 2. Frecuencia y porcentaje por dimensiones

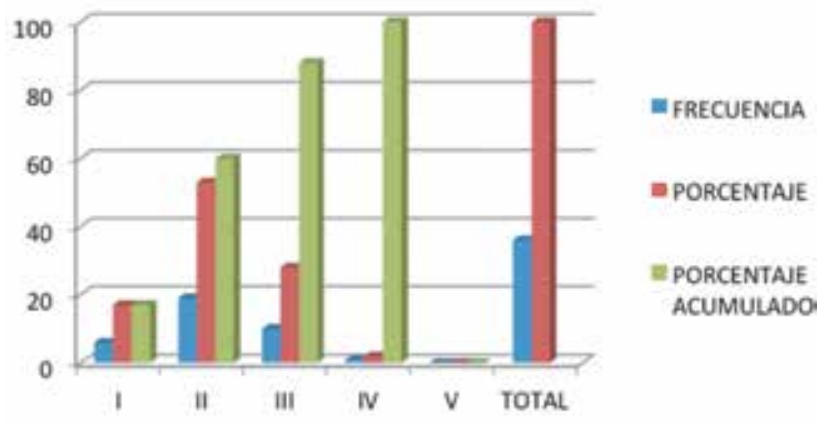

El $88 \%$ de los estudiantes a quienes se les aplicó la prueba se encuentran dentro del segundo y tercer nivel que corresponde a la dimensión relacional intertextual y enunciativa, respectivamente, lo cual implica que estos estudiantes presentan dificultades para identificar situaciones discursivas, el uso condicionado del código, la caracterización de quienes participan en dicha situación y la identificación del 
propósito comunicativo, así como problemas para determinar la intención que tiene el enunciador de acuerdo con la audiencia a la que se dirige. Por lo tanto, si los estudiantes aún no han superado estas etapas en la comprensión lectora, tampoco podrán identificar elementos relacionados con niveles de intertextualidad o reconocimiento de aspectos socioculturales inherentes al texto. En consecuencia, no han desarrollado ninguna de las dimensiones estrictamente críticas que se encuentran ubicadas en los niveles IV y V.

Por otro lado, no deja de ser preocupante que el $17 \%$ de la población objeto de estudio se encuentra ubicada en el nivel I, es decir, apenas realiza una tarea cognitiva de apropiación literal o inferencial del texto a través de la cual escasamente se acerca al conocimiento superficial y global del texto. Solo el $2 \%$ de los estudiantes alcanzaron dimensiones de competencia lectora propiamente críticas, esto es: valorativa y sociocultural.

Así que es necesario diseñar e implementar desde la universidad estrategias para fortalecer las dimensiones que configuran la competencia en lectura crítica, específicamente las relacionadas con las dimensiones valorativa y sociocultural, de tal manera que los estudiantes que ingresan a I o a V semestre (por convenio con escuelas normales) del programa, cuando lleguen a los últimos semestres puedan obtener un buen desempeño en la prueba Saber Pro, pero sobre todo, que como futuros docentes con profundización en Lengua Castellana, se caractericen por ser lectores críticos, reflexivos y analíticos, no solo de los textos que leen sino del mundo que los rodea.

En este contexto, los docentes de educación superior, conscientes de estas deficiencias de lectura, deben iniciar por desarrollar pensamiento crítico en sus estudiantes, entendido este como "un compromiso intelectual y ético que insiste en los estándares y criterios mediante los cuales se diferencia de un pensamiento acrítico (Lipman, 1991). En este sentido, la formación del pensamiento crítico en la educación superior se fortalecerá en los estudiantes siempre y cuando se incremente la cantidad y calidad de significados que los estudiantes obtengan a partir de lo que leen, escriben, hablen, escuchen y perciben. Por lo tanto, la actividad docente en el aula debe caracterizarse por guiar a sus estudiantes hacia la comprensión del texto, la formulación de inferencias, el análisis y resolución de problemas, la toma de decisiones y el cálculo de probabilidades; es decir, propender por el dominio de las habilidades del pensamiento.

En este sentido, la lectura como principal vía para acceder a la cultura y al pensamiento humano debe constituirse en un elemento esencial en la formación integral de cualquier área del conocimiento. Es así como la universidad debe fomentar a través de la investigación y de sus prácticas cotidianas, el cultivo de la lectura crítica y con ella el desarrollo del pensamiento crítico, como algo imprescindible para toda persona que se esté preparando para ejercer con madurez y responsabilidad una profesión. $Y$ es que la lectura crítica abre espacios para que el estudiante asuma posturas éticas con una mente abierta para comprender y analizar con diferentes miradas la realidad que lo rodea. Es necesario ayudar a los estudiantes a que vean diferentes ángulos de un mismo asunto o problema, a que tengan frente a las dificultades o los inconvenientes de la vida, no la actitud del que espera el mandato o la consigna para obedecerla ciegamente, sino que puedan deliberar y enfrentar las diversas encrucijadas morales que tendrán a lo largo de su vida (Vásquez, 2010).

Por lo tanto, frente a cada lectura el estudiante deberá asumir una postura distinta y dejará salir de sí mismo todo su interés, conocimiento y disposición para trabajarlo y ser trabajado por él, de tal modo que se debe acostumbrar a los estudiantes a cuestionarse, asombrarse, a indagar dentro de un contexto en que priman los 
discursos especializados de las distintas profesiones; tal como lo plantea Freire:

la lectura del mundo precede a la lectura de la palabra, de allí que la posterior lectura de esta no pueda prescindir de la continuidad de la lectura de aquel. Lenguaje y realidad se vinculan dinámicamente. La comprensión del texto al ser alcanzada por su lectura crítica, implica la percepción de relaciones entre el texto y el contexto $(2004$, p. 125$)$.

\section{b. Análisis dimensiones y competencias en lectura crítica}

\section{- Dimensión textual}

Teniendo en cuenta que la dimensión textual hace referencia a la forma como se rastrea y articula la información de un texto, con el fin de comprender su contenido, en el test diagnóstico se hicieron preguntas relacionadas con el reconocimiento del tema general de la lectura y la comprensión de significados de palabras y expresiones que aparecen explícitamente en el texto (dos preguntas: 1 y 10). Este primer paso es esencial en el desarrollo del pensamiento crítico de la persona, por cuanto le permite tener un amplio y claro conocimiento de la información del texto con el que evidentemente más adelante podrá hacer argumentaciones válidas sobre su posición frente a diferentes aspectos.

Por otro lado, es importante destacar que para los estudiantes es más fácil identificar la temática de un texto, ya que el $66 \%$ contestó adecuadamente y les es más difícil comprender el significado de algunas expresiones o palabras que se encontraban en el texto ya que solo el 34\% lo hizo en forma acertada, permitiendo deducir que existe una carencia léxica en el lector que le impide realizar un adecuado proceso de comprensión.

\section{- Dimensión intertextual}

En la dimensión intertextual se hace un avance hacia el reconocimiento de las relaciones que se establecen, tanto sintáctica como semánticamente, en un texto o entre dos textos. En este caso, se formuló una pregunta (5) para que el estudiante estableciera relación semántica entre el pensamiento del autor con las afirmaciones de un personaje que menciona en el texto, más explícitamente el escritor Mario Vargas Llosa. Para lograr este fin, es necesario que el lector haga una lectura minuciosa que le facilite realizar conexiones entre varias ideas que aparecen dentro del texto. Teniendo en cuenta que el $46 \%$ de los estudiantes contestaron correctamente la pregunta, se hace necesario implementar estrategias que lleven al desarrollo de una competencia reflexiva en los estudiantes, para que lo caractericen como una persona que puede enfrentar un texto en forma crítica, en el que no solamente ponga en juego habilidades de pensamiento como el análisis y la comprensión de la información, sino que participe como evaluador de argumentos, pueda presentar conjeturas, supuestos, inferir implicaciones, reconocer estrategias argumentativas, entre otras.

\section{- Dimensión enunciativa}

Para esta dimensión, que es necesaria para continuar con la comprensión del texto global y preparar a los estudiantes para las dimensiones y competencias estrictamente críticas, además que se avanza en la profundidad del textos, se preguntó a los estudiantes por el tono que predomina en el texto (pregunta 6), por cuanto en esta dimensión se indaga por el propósito comunicativo y la intención del autor de acuerdo con la audiencia a la que va dirigido, así mismo, cómo el lenguaje empleado por el autor da cuenta de su saber o de sus creencias, expresando verdades, deseos, certidumbres, reflexiones, dudas, entre otras experiencias de vida. Las opciones interrogaban si el tono predominante del discurso era reivindicador, conmiserativo, reflexivo o alentador.

El $66 \%$ de los encuestados escogieron la respuesta correcta. Lo anterior demuestra que una gran mayoría de los estudiantes objeto de es- 
tudio han avanzado a niveles de interpretación más profunda del texto, pero un número significativo de ellos $34 \%$, aún permanecen en las dimensiones textual e intertextual, solo ubican y reconocen información presente en el acto comunicativo, lo que hace más difícil su proximidad a niveles críticos de la lectura.

\section{- Dimensión valorativa}

Tres preguntas se formularon con respecto a esta dimensión, que es propiamente crítica por cuanto se indagó por los componentes ideológicos que configuran el texto, de tal manera que el estudiante pudiera demostrar una visión crítica de este, a través de apreciaciones de tipo valorativo acerca de las condiciones sociales, históricas, políticas y culturales que le subyacen. En esta dimensión crítica el estudiante va más allá de lo evidente, identificando las opiniones del autor, los argumentos que la sustentan y en general todos aquellos elementos que están detrás del texto, por lo que requiere del reconocimiento de contenidos valorativos e ideológicos.

Para evaluar esta dimensión, la prueba presentaba preguntas relacionadas con la opinión del autor, sus argumentos para sustentarla, las pretensiones al emitir juicios valorativos y las relaciones que se establecen con otros textos de la cultura y que dan sustento al contenido críticovalorativo (2, 3 y 4). Una vez analizadas las respuestas se pudo inferir que a la mayoría de estudiantes evaluados les cuesta trabajo reconocer las perspectivas y puntos de vista que circulan en el texto, lo cual evidencia que no es fácil pasar a este nivel, por cuanto los estudiantes no poseen en su mayoría la capacidad de reconocer aspectos sintácticos y semánticos que conforman el texto, ni la competencia para identificar y reconstruir las subjetividades del autor.

\section{- Dimensión sociocultural}

En esta dimensión, la segunda propiamente crítica, se reconocen aspectos que tienen relación con la cultura y la sociedad, así como la forma en que el discurso es un mediador entre los procesos comunicativos inherentes al ser humano, actor fundamental que comprende e interpreta la realidad de la cual surge dicho discurso y la incidencia ética, moral y social que se le atribuye a la palabra para reflejar el contexto en el que se desenvuelve el individuo.

Con base en lo anterior, la prueba aplicada, incluía aspectos relacionados con la importancia de reconocer la intención y el propósito que orientan al autor del texto en la puesta en escena de este. Así mismo, el lector pone en juego sus saberes previos de tal manera que comprenda la realidad de la cual surge el discurso. Las preguntas de esta dimensión $(7,8$ y 9$)$ conducen a hacer reflexionar al lector sobre una acción específica en este caso, recomendar a aquellas mujeres impuntuales, que cambien su actitud, ya que están incidiendo en forma negativa en la productividad de una empresa.

Por otro lado, estas preguntas se encuentran directamente relacionadas con la competencia reflexiva de la lectura crítica, por cuanto exige reconocer y validar los juicios y argumentos que soportan la tesis del autor. Las respuestas de los estudiantes permiten deducir que aún les falta desarrollar este nivel de competencia, puesto que este tipo de preguntas requieren por parte del estudiante un buen nivel de pensamiento críticoreflexivo que le permita trascender el texto.

En consecuencia se hace necesaria la implementación de estrategias que fortalezcan las competencias de lectura crítica en el aula, con miras al mejoramiento de la calidad de la educación de los estudiantes del programa, y que se verá reflejado positivamente en su futuro desempeño laboral. Para tal fin, es vital involucrar diversidad de textos como periódicos, revistas, ensayos, crónicas, artículo, entre otros en todas las áreas de la licenciatura; así mismo, lograr una capacitación pedagógica de los docentes, orientada a garantizar la formación de lectores críticos que se desempeñarán asertivamente como ciudadanos con criterio. 
Es importante resaltar que las competencias en lectura crítica están estrechamente relacionadas con las dimensiones evaluadas en las pruebas Saber Pro, por cuanto las dimensiones textuales evidentes y relacionales intertextuales se correlacionan con la competencia de identificar y entender los contenidos locales que conforman un texto. La competencia de comprender cómo se articulan las partes de un texto para darle sentido global se configura dentro de la dimensión enunciativa, ya que establece relaciones semánticas en el texto. La competencia de poder reflexionar a partir de un texto, requiere el desarrollo de las dimensiones valorativas y socio cultural, puesto que se trasciende a situaciones del entorno del lector y el escritor. Así que el fortalecimiento de las competencias en lectura crítica exige del docente el conocimiento de las implicaciones presentes en cada una de las dimensiones de lectura crítica.

\section{Conclusiones}

El mayor porcentaje de los estudiantes a quienes se les aplicó la prueba, se encuentran dentro del segundo y tercer nivel que corresponde a la dimensión relacional intertextual y enunciativa, es decir, han desarrollado las competencia de identificar y entender los contenidos locales que conforman un texto, así como la de comprender cómo se articulan las partes de un texto para darle sentido global.

Algunos estudiantes de VI semestre presentan problemas para identificar situaciones discursivas, reconocer las características de quienes participan en dichas situaciones, igualmente tienen dificultad en cuanto al reconocimiento del propósito comunicativo del texto. Así mismo, no han desarrollado la habilidad suficiente oopara determinar la intención que tiene el enunciador de acuerdo con la audiencia a la que se dirige.

Es preocupante para las docentes investigadoras, el hecho de que estudiantes de VI semestre, que se están formando para ser docentes de Lengua Castellana, se encuentren ubicados en el nivel I de comprensión textual, es decir, apenas realizan una tarea cognitiva de apropiación literal e inferencial del texto. Solo un pequeño porcentaje de los estudiantes evaluados, alcanzaron dimensiones de competencia lectora propiamente críticas, esto es: valorativa y sociocultural.

El desarrollo de competencias de lectura crítica en los estudiantes está estrechamente relacionada con el dominio de las dimensiones; textual evidente, relacional intertextual, enunciativa, valorativa y socio cultural.

Por lo anterior, las universidades en especial la UPTC debe promover el pensamiento crítico en los estudiantes, con el fin de lograr profesionales integrales y competentes para liderar procesos de cambio favorables en la sociedad, en especial cuando se trata de docentes en formación, quienes tenderán bajo su responsabilidad la orientación educativa y social de los niños y jóvenes de nuestro país.

En la actualidad se reconoce la necesidad de desarrollar habilidades del pensamiento crítico en los estudiantes para que sean competentes y así asumir desde diferentes miradas y ópticas los textos académicos involucrados en cada una de las áreas del saber, a través de la implementación de estrategias que permitan el diálogo y la discusión académica.

Los docentes estamos llamados a asumir una actitud de cambio que permita desarrollar en nuestros estudiantes la capacidad de asombro frente al discurso, que los textos están a su alcance no solo para ser interpretados sino también para ser valorados, disfrutados, criticados y por qué no, superados.

A partir del presente estudio, las investigadoras quieren abrir el camino a la reflexión acerca de cómo se han llevado a cabo las prácticas lectoras en el aula, para proponer alternativas de solución transversales a todas las áreas que permitan la formación de verdaderos lectores críticos. 


\section{UNIVERSIDAD PEDAGÓGICA Y TECNOLÓGICA DE COLOMBIA FACULTAD DE ESTUDIOS A DISTANCIA FESAD ESCUELA DE CIENCIAS HUMANÍSTICAS Y DE EDUCACIÓN LICENCIATURA EN EDUCACIÓN BÁSICA}

\section{PRUEBA DIAGNÓSTICA LECTURA CRÍTICA}

OBJETIVO: Identificar el nivel de lectura crítica que tienen los estudiantes del programa de Educación Básica de la FESAD-UPTC.

Instrucciones: A continuación encontrará dos textos con preguntas de Opción Múltiple, después de leerlos, responda a las preguntas haciendo uso de la hoja de respuestas.

\section{TEXTO 1: Preguntas 1 a 6}

Por muchos años, hemos creído que el Perú es un mendigo sentado en un banco de oro, pensando que la riqueza estaba en nuestros recursos naturales. Siendo nuestro país el principal productor mundial de páprika y de lana de alpaca, el principal exportador de espárragos, harina de pescado y plata, y el segundo en zinc y castañas; teniendo más de 25 mil especies de plantas, y estando considerado como la cuarta nación con mayor biodiversidad del mundo, es fácil imaginarnos ese banco de oro que hemos heredado. Y es esa interpretación la que nos hace creer que son las dotes naturales las que "nos han tocado" y que únicamente ellas nos garantizan el progreso; es decir, lo que vale, lo que marca la diferencia es lo que uno trae consigo. Sin embargo, ¿es eso cierto?, ¿el éxito es fruto de la suerte o del esfuerzo?, ¿la clave está en la herencia o en la iniciativa y el emprendimiento?, ¿un genio nace o se hace? Mario Vargas Llosa nos enseña que no bastan las dotes y el talento con los que nacemos. Un genio se hace con disciplina, pasión y esfuerzo.

Ser escritor, enseña Mario, es un trabajo como cualquier otro que, aunque dependa en gran medida de la creatividad y la imaginación, requiere de disciplina y no de esperar que buenamente llegue la inspiración. Si se ve como un trabajo, y ponerse reglas y controles es clave para lograr el objetivo. Así, más importante que lo que uno tiene es qué hace con lo que le ha tocado. Y eso es un tema de voluntad y perseverancia, de motivación y ánimo para conseguir los objetivos y metas que uno se propone, venciendo las dificultades que puedan surgir en el camino.

Por eso, creernos un país de inmensas pero pobre es suponer que tenemos que encontrar tesoros materiales para hacernos ricos, cuando, más bien, la riqueza está en nosotros mismos, en nuestra forma de ser, nuestra capacidad de superación y emprendimiento, nuestra creatividad, laboriosidad y fuerza cultural; en suma, en nuestras actitudes; y es allí donde debemos buscar el desarrollo de nuestro país.

1. ¿Cuál es el tema del texto anterior?

A. Una discusión sobre los factores que impiden el desarrollo de un país, según Vargas Llosa.

B. Las cualidades personales de Mario Vargas Llosa como ejemplo de la riqueza nacional.

C. Los elementos que garantizan el éxito en las personas.

D. La actitud de las personas como elementos del progreso del país.

2. ¿Cuál es la opinión del autor en el texto anterior?

A. Solo el desarrollo de una actitud ganadora hará salir al país del subdesarrollo.

B. Es falso que el Perú sea un mendigo sentado en un banco de oro, pues las verdaderas carencias de los peruanos no se hallan en sus actitudes.

C. El Perú es un país de inmensas riquezas pero pobre debido a la falta de disciplina de su población.

D. No son las riquezas o bienes innatos la garantía del progreso del país, sino las actitudes de su gente.

3. ¿Cuál es el argumento principal que desarrolla el autor del texto anterior para sostener su posición?

A. La motivación y el ánimo son centrales para alcanzar el desarrollo.

B. Más importante que tener riquezas es saber qué actitudes desarrollar con ellas.

C. Nuestra creatividad, laboriosidad y fuerza cultural son un ejemplo para otros.

D. El ahínco de la gente es lo que, finalmente, garantiza el desarrollo.

4. ¿Cuál es la síntesis de la argumentación del texto anterior?

A. El desarrollo de un país no reside en los bienes interiores o heredados sino en las actitudes de su población, ya que, más que la riqueza, importa lo que uno haga con ella.

B. El éxito de un país no está en la suerte o en la herencia, sino en la valoración de sus recursos materiales y humanos.

C. Solo el que sabe qué hacer con sus riquezas es capaz de generar un verdadero desarrollo en el país.

D. Debido a que la gente es el principal motor del desarrollo, se debe entender que el progreso solo lo genera la variedad de recursos con los que cuenta un país. 
5. A partir del texto anterior, se puede afirmar que:

I. Mario Vargas Llosa ha logrado el Nobel solo por la gran capacidad de trabajo que ha mantenido durante todos estos años.

II. Todo trabajo es el resultado de la voluntad y la perseverancia.

III. Solo la actitud asegura el desarrollo de un país.
A. SoloI
B. Solo Iy II
C. Solo II y III
D. Ninguna

6. El tono que predomina en el texto anterior es:

A. Reivindicador

B. Conmiserativo

C. Reflexivo

D. Alentador

\section{TEXTO 2: Preguntas 7 a 9}

Las mujeres tenemos fama de ser impuntuales, aunque esto no debería ser considerado como una regla. Ahora bien, si usted pertenece al grupo de las que llegan tarde a todas partes, es conveniente que enmiende de una vez este mal hábito, porque el tiempo posee un valor extraordinario, sobre todo para las personas que trabajan en una empresa. Se sorprendería al saber cuánto gana un alto ejecutivo en solo minutos. Si está citada para una reunión de trabajo a las ocho de la mañana, ino llegue a las ocho y cinco minutos! En ese lapso que la esperan, se puede haber redactado algún informe o realizado avances de negocio. Además de hacer perder el tiempo a otros, la imagen de irresponsable y poco profesional que proyecta podría hacer pensar que usted no es la persona idónea para el cargo que ocupa. Si tiene que retrasarse, llame y avise con anticipación.

\section{7. ¿Cuál es la intención de la autora del texto?}

A. Llamar la atención sobre la impuntualidad de las ejecutivas.

B. Explicar la importancia del tiempo para los hombres que son ejecutivos.

C. Recomendar a las mujeres impuntuales que se corrijan.

D. Aconsejar a las ejecutivas que deben el tiempo de los otros.

\section{8. ¿Cuál es la tesis del texto?}

A. La impuntualidad femenina es famosa.

B. Es una mala imagen la que proyecta una mujer impuntual, porque el tiempo es muy valioso en una empresa.

C. El tiempo es un valor muy reconocido en el ambiente profesional, por lo que una trabajadora no debe ignorarlo.

D. Las mujeres que son impuntuales deben dejar de serlo.

\section{Seleccione la alternativa que resuma la propuesta de la autora}

A. La puntualidad es muy apreciada en el ambiente empresarial, porque permite ganar dinero.

B. La mujer habituada a la impuntualidad debe corregir este defecto, pues el tiempo es muy valioso, sobre todo en una empresa.

C. La buena imagen de un trabajador se ve afectada por la impuntualidad, de modo que debe procurar llegar más temprano.

D. El tiempo en los ambientes empresariales es considerado dinero, y las trabajadoras deben respetarlo.

10. ¿Qué sentido tiene la palabra "enmiende" en el texto?

a. Analizar

b. Arreglar

c. Evaluar

d. Solucionar

Fuente: Ejercicio de comprensión lectora crítica para examen de admisión Universidad Católica, 2014. Se realizaron algunos cambios.

Muchas gracias por su colaboración

Las investigadoras.

Grupo SIEK

FESAD-UPTC 


\section{Referencias bibliográficas}

Ander-Egg, E. (1984). Metodologías de acción social. España: Universidad de Jaen.

Cassany, D. (2006).Tras las líneas. Sobre la lectura contemporánea. Barcelona: Anagrama.

Cooper J., D. (1990) citado por Maqueo (2006). Cómo mejorar la comprensión lectora. Madrid: Visor.

Freire (1989). La alfabetización como elemento de formación de la ciudadanía. En Política y educación, XXI, 5056. México: Siglo XX.

Giroux, H. (1992). Teachers as intellectuals. Towards a critical pedagogy of learning. Massachusetts: Bergin \& Garvey. Versión española: Los profesores como intelectuales. Hacia una pedagogía crítica del aprendizaje. Barcelona: Paidós.

ICFES (2014). Módulo de lectura crítica. Saber Pro 20141. Recuperado de file:///C:/Users/ECHE/Downloads/ Lectura\%20critica\%202013\%202.pdf (14 de febrero de 2015).

ICFES (2014). Módulo de lectura crítica. Saber Pro 20142. Recuperado de file:///C:/Users/ECHE/Downloads/ Lectura\%20critica\%202013\%202\%20(1).pdf (14 de febrero de 2015).

Kanpool, B. (1994). Critical pedagogy: An introduction. Londres: Bergin \& Garvey.

Klooster, D. (2001). What is critical thinking?, thinking classroom. A Journal of Reading, Writing and Critical Reflection, 4, 36-40. Primavera.

Lemos, C., Cuello, E., Roys, N., y Rojas, L. (2004). Análisis de los resultados de la prueba Saber Pro. Programa de Licenciatura en Etnoeducación. Universidad de la Guajira.
Lipman, M. (1991). Pensamiento complejo y educación. Madrid: Ediciones de la Torre.

Maya, L, (2011). Una práctica de lectura crítica con los estudiantes de la Universidad de Medellín. Revista Virtual Universidad Católica del Norte, 34, 162-193.

Maqueo, A. M. (2006). Lengua, aprendizaje y enseñanza: El enfoque comunicativo de la teoría a la práctica. Bogotá: Editorial Limusa.

MEN (1990). Lineamientos curriculares de Lengua Castellana.

Ministerio de Educación Nacional (1990). Lineamientos Curriculares Lengua Castellana. Bogotá: Magisterio.

Rodríguez, A. N. (sep.-dic., 2007). Lectura crítica y escritura significativa. Acercamiento didáctico desde la lingüística. Laurus Revista de Educación, 13(25). Recuperado de http://redalyc.uaemex.mx/src/inicio/ ArtPdfRed.jsp?iCve=76111479012 (15 de marzo de 2015).

Sampieri H., R. et al. (2010). Metodología de la investigación. (5a ed.). México: McGraw Hill.

Serafini, F. (2003). Informing our practice: transactional, and critical perspective on children's literature and reading instructions. On line: www.readingonline.org/ articles/art_index.asp?HERF=serafuni/index.html (25 de febrero de 2015).

van Dijk, T.A. (1989). Estructuras y funciones del discurso. México: Siglo XXI.

Vásquez, F. (enero, 2015). Repensar la lectura y la lectura crítica. En Ruta maestra. Competencias y ciudadanía, 10. Bogotá: Santillana. Recuperado de: http:// santillana.com.co/rutamaestra/revistas_pdf/ruta_ maestra_v_0010.pdf (12 de marzo de 2015). 